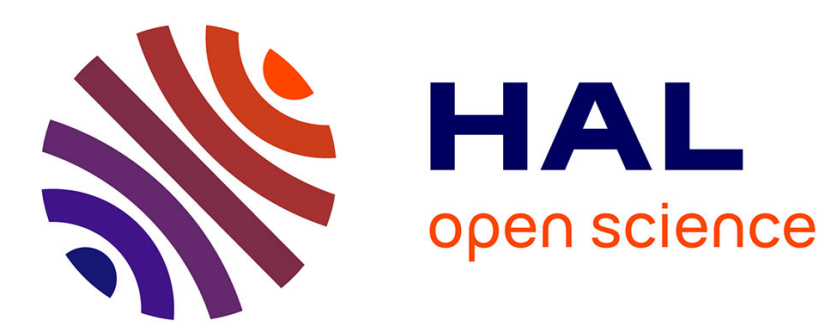

\title{
More pessimism than greediness: a characterization of monotone risk aversion in the Rank-Dependent Expected Utility model
}

\author{
Alain Chateauneuf, Michèle Cohen, Isaac Meilijson
}

\section{- To cite this version:}

Alain Chateauneuf, Michèle Cohen, Isaac Meilijson. More pessimism than greediness: a characterization of monotone risk aversion in the Rank-Dependent Expected Utility model. Economic Theory, 2005, 25, pp.649-667. 10.1007/s00199-003-0451-7 . halshs-00211906

\section{HAL Id: halshs-00211906 \\ https://shs.hal.science/halshs-00211906}

Submitted on 22 Jan 2008

HAL is a multi-disciplinary open access archive for the deposit and dissemination of scientific research documents, whether they are published or not. The documents may come from teaching and research institutions in France or abroad, or from public or private research centers.
L'archive ouverte pluridisciplinaire HAL, est destinée au dépôt et à la diffusion de documents scientifiques de niveau recherche, publiés ou non, émanant des établissements d'enseignement et de recherche français ou étrangers, des laboratoires publics ou privés. 
More pessimism than greediness: a characterization of monotone risk aversion in the Rank-Dependent Expected Utility model ${ }^{\not}$

\author{
A lain Chateauneuf ${ }^{1}$, M ichale Cohen ${ }^{2}$ and I saac M eilijson ${ }^{3}$ \\ ${ }^{1}$ CERM SEM , Universit de Paris I, Maison des Sciences Economiques, 106-112 Bld de \\ I'Hopital, 76475 Paris, France (email: chateaun@univ-paris1.fr) \\ 2 EUREQua, Universit de Paris I, M aison des Sciences Economiques, 106-112 Bld de \\ I'Hopital, 76475 Paris, France (email: cohenmd@univ-paris1.fr) \\ 3 School of M athematical Sciences, Raymond and Beverly Sackler Faculty of Exact Sciences, \\ Tel Aviv University, Tel A viv 69978, I srael (e-mail: meilijson@math.tau.ac.il)
}

Summary. This paper studies monotone risk aversion, the aversion to monotone, meanpreserving increase in risk (Q uiggin [21]), in the R ank Dependent Expected Utility (RDEU) model. This model replaces expected utility by another functional, characterized by two functions, a utility function $u$ in conjunction with a probability-perception function $f$. $M$ onotone mean-preserving increases in risk are closely related to the notion of comparative dispersion introduced by Bickel \& Lehmann $[3,4]$ in Non-parametric Statistics. We present a characterization of the pairs $(u ; f)$ of monotone risk averse decision makers, based on an index of greediness $G_{u}$ of the utility function $u$ and an index of pessimism $P_{f}$ of the probability perception function $\mathrm{f}$ : the decision maker is monotone risk averse if and only if $P_{f}, G_{u}$. The index of greediness (non-concavity) of $u$ is the supremum of $\left.u q x\right)=19 y$ ) taken over $y \cdot x$. The index of pessimism of $f$ is the in ${ }^{-}$mum of $\frac{l_{i} f(v)}{1_{i} v}=\frac{f(v)}{v}$ taken over $0<v<1$. Thus, $G_{u}, 1$, with $G_{u}=1 i \circledast u$ is concave. If $P_{f}, G_{u}$ then $P_{f}, 1$, i.e., $f$ is majorized by the identity function. Since $P_{f}=1$ for Expected Utility maximizers, $P_{f}, G_{u}$ forces $u$ to be concave in this case; thus, the characterization of risk aversion as $P_{f}, G_{u}$ is a direct generalization from EU to RDEU. A novel element is that concavity of $u$ is not necessary. In fact, $u$ must be concave only if $P_{f}=1$.

K eywords and P hrases: Risk aversion, pessimism, greediness, Rank-dependent Expected Utility.

J EL Classi ${ }^{-}$cation Numbers: D81

\footnotetext{
We are most grateful to Mark Machina, Peter Wakker and two anonymous referees for very helpful suggestions and comments.

Correspondence to: $M$. Cohen
} 


\section{Introduction}

Under the expected utility (EU) model, a decision maker (DM) is characterized by a single function $u$, called the utility function. The crucial criticism of the EU model by Allais [1] from a theoretical point of view is that in such a model, the same function $u$ characterizes two distinct behaviors - satisfaction from certain wealth and attitude to risk - that have no reason to be linked: a DM with diminishing marginal utility on certain wealth may be risk-seeking, but this is precluded by the $\mathrm{EU}$ model.

The rank-dependent expected utility (RDEU) model (see [20, 32, 11, 28, 21, 6]) has been built in part as an attempt to answer the criticism of Allais. A RDEU DM is characterized by two functions: a utility function $u$ on outcomes and a probability-perception function $f:[0 ; 1]$ ! [0; 1]. A RDEU decision maker compares a lottery with cumulative distribution function $F$ with another by the expected utility of the lottery evaluated as if the lottery had distribution function 1 i $f(1 ; F)$ instead of $F$ (see (4)). Thus, $f$ below (above) the identity function indicates pessimism (optimism), while the diagonal case $f(p)=p$ is the perception-neutral attitude of EU maximizers.

A risk averse DM is usually de- ned as one that, for every bounded random variable, prefers the expectation of the random variable to the random variable itself. This notion will be called weak risk aversion. A version to mean-preserving increase in risk (MPIR) in the sense of Rothschild \& Stiglitz (see [14, 24]) will be called strong risk aversion.

In Yaari's Dual Theory (RDEU with linear $u$ ), weak risk aversion is characterized by $f$ majorized by the identity function (henceforth pessimism). Monotone mean-preserving increase in risk (M-MPIR), introduced by Quiggin $[20,21]$ and properly de- ned in the sequel, was then obtained ([5,9], implicitly in [32]) as the kind of added risk to which weakly risk averse Dual Theory decision makers are averse.

It is tautologically true that strong risk aversion implies monotone risk aversion, which implies weak risk aversion. In the EU model, weak risk aversion is characterized by concavity of $u$. MPIR was then obtained $([14,24])$ mathematically as the kind of added risk to which weakly risk averse EU decision makers are averse. Thus, in EU theory, weak, monotone and strong risk aversion are equivalent. In Dual Theory, weak and monotone risk aversion are equivalent, while strong risk aversion requires $f$ to be convex ([32]).

The situation in the RDEU model is more ${ }^{\circ}$ exible due to the trade-o ${ }^{\circ}$ between risk aversion implications of utility and probability perception. However, if the utility on 
outcomes $\mathrm{u}$ is concave, the situation is much like for the Dual Theory. Weak risk aversion under all concave utilities $u$ is characterized by perception functions displaying pessimism ( see [21, 32]), while strong risk aversion under all concave utilities u requires the stricter condition that $f$ be convex (see Chew, Karni \& Safra [11]). Chew, Karni \& Safra [11] proved, more generally, that concavity of $u$ and convexity of $f$ are necessary and su \pm cient for strong risk aversion. Quiggin $[20,21]$ also brought to light that M-MPIR is the kind of increase in risk to which RDEU weakly risk averse decision makers with concave utility (not only linear) are averse.

Similarly to the de ${ }^{-}$nition where $G$ is a MPIR with respect to $F$ if there exist random variables $X$ and $Z$ with $X$ distributed $F, X+Z$ distributed $G$ and $Z$ is "noise" around $X(E[Z j X]=0$ a.s.), the notion of $G$ being a $M-M P I R$ requires instead that $Z$, with mean zero, be comonotone with $X$. B ecause $\mathrm{EU}$ (under all concave utilities) is a special case of RDEU (under all concave utilities and convex probability perception functions), M-MPIR is in particular MPIR.

The notion of monotone risk aversion is model-free; it has been proved to be useful in EU (see Section 2.3) and is well ${ }^{-}$tted to RDEU theory (see $[11,20,28,32]$ ), where comonotonicity plays a fundamental part at the axiomatic level. The above analysis restricted $u$ to the case of concave functions. Consistent with Allais' criticism, it is of interest to study whether a decision maker can be averse to risk without $u$ being concave. In a previous paper, Chateauneuf $\&$ Cohen [7] proved that pessimism of $f$ is a necessary condition for weak risk aversion, while concavity of $u$ is not, but did not succeed to fully characterize weak risk aversion. This is the subject of our current research, by which we know that when non-concave $u$ are allowed, weak risk aversion does not imply monotone risk aversion.

In this paper we characterize the class of pairs $(u ; f)$ of utility and probability perception function that model aversion to M-MPIR.

More speci ${ }^{-}$cally, for an increasing probability perception function $f$, let

$$
P_{f}=\inf _{0<v<1}\left[\frac{1 ; f(v)}{1 ; v}=\frac{f(v)}{v}\right]
$$

be its index of pessimism (see Section 3.1). The probability perception function displays pessimism, i.e., is majorized by the identity function, if and only if $P_{f}, 1$. 
For a strictly increasing utility function $u, \mathrm{de}^{-}$ne

$$
\mathrm{G}_{u}=\sup _{\mathrm{x}_{1}<\mathrm{x}_{2} \cdot \mathrm{x}_{3}<\mathrm{x}_{4}}\left[\frac{\mathrm{u}\left(\mathrm{x}_{4}\right) \mathrm{i} \mathrm{u}\left(\mathrm{x}_{3}\right)}{\mathrm{x}_{4} \mathrm{i} \mathrm{x}_{3}}=\frac{\mathrm{u}\left(\mathrm{x}_{2}\right) \mathrm{i} \mathrm{u}\left(\mathrm{x}_{1}\right)}{\mathrm{x}_{2} \mathrm{i} \mathrm{x}_{1}}\right]
$$

to be its index of non-concavity. This index (also called greediness, see Section 3.2) satis ${ }^{-}$es $G_{u}, 1$ as well, and the value 1 corresponds exclusively to concavity. When $u$ is di Rerentiable, it is the supremal value of $u^{9}(x)=u^{9}(y)$ taken over $x, y$.

The characterization of monotone risk aversion in the RDEU model will be based on the comparison of these two indices.

In Section 2 we recall the basic de nitions and some basic properties of M-MPIR and monotone risk aversion, and justify the interest in M-MPIR, irrespective of the model of decision under risk used. The corresponding introductory material on the RDEU model is contained in Section 3. Section 4 states the main result (Theorem 1) characterizing monotone risk aversion in the RDEU model and its corollary that handles the case of monotone risk-seeking attitudes, illustrates in the RDEU context the monotone risk seeking behavior of a D M with diminishing marginal utility and compares monotone and strong risk averse attitudes.

\section{M onotone mean-preserving increase in risk and monotone risk aversion}

\subsection{Notation}

We assume that risk prevails and describe it through a set of states of nature - $=[0 ; 1]$ endowed with the uniform probability measure $P$ on the $B$ ore $3 / 4^{-}$eld. Let $V=f X ; Y ; \varnothing \varnothing \varnothing$ be the set of bounded $\mathrm{R}$-valued random variables on - .

For any $X$ in $V$, we denote by $F_{X}$ (respectively, $\bar{F}_{X}=1 ; F_{X}$ ) the cumulative (decumulative) distribution function of $X$ and by $E(X)$ the expected value of $X$. Let $D$ be the set of cumulative probability distribution functions on $\mathrm{R}$.

The distribution (or law) of a discrete random variable $\mathbf{Z}$ will be denoted by

$$
L(Z)=\left(x_{1} ; p_{1} ;:: ; x_{k} ; p_{k} ;:: ; x_{n} ; p_{n}\right)
$$

with $x_{1}<x_{2}<\Phi థ \varnothing<x_{n}, p_{i}, 0$ and ${ }^{P} p=1$.

\subsection{Comonotonicity and monotone mean-preserving increase in risk}

T wo functions $X$ and Z: - ! R are comonotone if each is non-decreasing in the other. Z may be non-decreasing in $X$ : - ! $R$, that is, $X(!)>X(! 9$ implies that $Z(!), Z(! 9$, without 
$Z$ being a function of $X$ at all, that is, without the further requirement that $X(!)=X(!)$ imply $Z($ !) $=Z$ (! 9. If $Z$ is non-decreasing in $X$ then $X$ is clearly non-decreasing in $Z$ as well, so this notion is symmetric ${ }^{1}$. The common formal de nition (see $[26,27,32,13]$ ) of this notion of functions that are non-decreasing in each other, or comonotone functions, is:

De $e^{-}$nition 1 Two real-valued functions $X$ and $Z$ on - are comonotone if for any ! and $!^{0} 2-,[X(!)$ i X (! 9][Z(!) i Z(! 9], 0.

Comonotonicity is not a transitive relation because constant functions are comonotone with any function. Consistent with the usual conventions, random variables are said to be comonotone if they are comonotone functions on some sure event.

Quiggin [21] introduced the following notion of monotone mean-preserving increase in risk (M-MPIR).

$\mathrm{De}^{-}$nition 2 For $\mathrm{X} ; \mathrm{Y} 2 \mathrm{~V}, \mathrm{Y}$ is a monotone mean-preserving increase in risk M-MPIR of $X$ if there exists a random variable $Z 2 V$ with $E(Z)=0$ such that $X$ and $Z$ are comonotone and $Y$ has the same probability distribution as $X+Z$. Equivalently, $X$ will be said to be a monotone mean-preserving reduction in risk of $Y$.

This is a very intuitive notion of increase in risk: $Y$ could be said to be a "progressive stretch" of $X$ : if an $X$-mass at $X$ is shifted to become a $Y$-mass at $y$, then all $X$-masses to the right of $\mathrm{x}$ will be shifted by at least $\mathrm{y} ; \mathrm{x}$. In particular, their distribution functions single cross. For illustrative intuition, observe that net income and tax are comonotone because each is a non-decreasing function of gross income. Thus, taxation (centered so as to display mean zero) accomplishes a monotone mean-preserving reduction in risk. The notion of price band stabilization, used in the theory of the ${ }^{-} \mathrm{rm}$, is al so a particular case of monotone reduction in risk.

Monotone increases or reductions in risk are assumed to be mean preserving. Except for this requirement, they coincide with the notion of comparative dispersion introduced in a statistical framework by Bickel \& Lehmann (see $[3,4])$ :

De $e^{-}$nition 3 For $F$ and $G$ in $D, F$ is Bickel \& Lehmann less dispersed than $G$ if for every $0<x<y<1 ; F^{1}{ }^{1}(y)$ i $F^{i}{ }^{1}(x) \cdot G^{1}(y)$ i $G^{1}{ }^{1}(x)$ :

\footnotetext{
${ }^{1}$ E quivalently, as some further thought will reveal, each of $X$ and $Z$ is a non-decreasing function of some third function from - to $R$, and this other function can always be taken to be $X+Z$.
} 
That is, all the interquantile intervals are shorter for $X$ than for $Y$. The de- nition of dispersion doesn't require $F$ nor $G$ to possess ' nite ${ }^{-}$rst moments, and even if they do, these moments need not be equal. In fact, $Y \gg G$ is more dispersed than $X \gg F$ if and only if $Y+c$ is more dispersed than $X$, for arbitrary c 2 R. Gathering di ®erent properties obtained in the literature on this subject ( see $[8,19,21]$ ), it is possible to obtain the following connection between the Bickel \& Lehmann dispersive order and M-MPIR .

Proposition 1 When two random variables $X$ and $Y$ in $V$ have the same expected value, $Y$ is a $M-M P I R$ of $X$ if and only if $F_{Y}$ is more dispersed than $F_{X}$ in the sense of Bickel \& Lehmann.

ॠrom now on, we concentrate on M-MPIR, the mean-preserving version of comparative dispersion and on the corresponding notion of attitude to risk, monotone risk aversion, to be viewed as aversion to M-MPIR. More formally:

De ${ }^{-}$nition 4 A DM is monotone risk averse (respectively, monotone risk seeking) if for any $X$ and $Y$ in $V$ with equal means such that $Y$ is a $M-M P I R$ of $X$, the DM weakly prefers $X$ to $Y$ (respectively, $Y$ to $X$ ).

2.3 M otivation for the study of monotone mean-preserving increase in risk and the dispersive order: insurance and portfolio management under the EU model

To gain some intuition on M-MPIR, note that, as a consequence of $\mathrm{De}^{-}$nition 3 , the distributions F and G must single cross. Thus (see Diamond \& Stiglitz [14]), G is a M PIR (in the sense of $\mathrm{R}$ othschild \& Stiglitz) with respect to $\mathrm{F}$. But De- nition 3 (i.e. dispersion) not only makes $F$ and $G$ single cross - it makes $G$ single cross every horizontal translation of $F$. This property is very meaningful for Insurance: a horizontal translation of $F$ is the distribution of a random variable $X_{i}$ c obtained from an $F$-distributed $X$ by the subtraction of an arbitrary constant c. This constant may play the role of insurance premium for an otherwise fair contract that replaces the distribution $G$ of the uninsured position $Y$ by the distribution $F$ of the random variable $X$. Since the distribution of $X ;$ c single crosses the distribution of $Y$ and the utility function $u$ is non-decreasing, the distributions of $u(X ; c)$ and $u(Y)$ single cross as well. Choosing the premium c so that $E(u(X ; c))=E(u(Y))$, $u(Y)$ becomes a MPIR with respect to $u(X ; c)$, so $E\left({ }^{\prime}(u(X ; c))\right), E\left({ }^{\prime}(u(Y))\right)$ for all non-decreasing and concave'. Since for EU DM's this characterizes $v\left(\phi={ }^{\prime}(u(\phi)\right.$ as 
displaying more risk aversion than $u$, this proves that a Arrow-P ratt more risk averse EU DM will always be ready to pay higher premiums for monotone mean-preserving reductions in risk. This property does not necessarily hold for standard mean-preserving reductions in risk, as shown by Ross [23] and others. This apparent drawback of the usual (A rrowPratt) notion of comparative risk aversion is in fact a drawback of the automatic use of second degre stochastic dominance as the reference for reduction of risk by insurance. Landsberger \& Meilijson [18] proved that M-MPIR is the weakest order compatible with the Arrow-Pratt index of risk aversion, in the class of all non-decreasing utility functions. J ewitt [15] introduced a weaker order, still stronger than Rothschild \& Stiglitz's, that characterizes risk reductions compatible with the Arrow-P ratt index in the class of nondecreasing concave utility functions. We respectfully credit J ewitt with the technical idea for connecting insurance premia with horizontal shifts of distributions. Landsberger \& M eilijson [19] have presented other applications of this stochastic order to insurance.

In the following sections we study a role played by monotone mean-preserving risk in the RDEU model. A forthcoming paper [9] analyzes other roles played by monotone risk and its weaker version Location-independent Risk by J ewitt, in the same model. We ${ }^{-}$nish this section on monotone risk by illustrating an application to Portfolio management.

In the standard problem of designing an optimal portfolio based on one safe and one risky asset, a natural prediction is that if the risky asset becomes riskier (in the usual R othschild \& Stiglitz (1970) sense, see [24]), the EU strongly risk averse investors will want less of it. This prediction was shown to be wrong by Rothschild \& Stiglitz in their 1971 paper (see [25]), where they present the following necessary and su \pm cient condition on the utility function $u$ under which a MPIR will always lead to a reduction in the allocation to the risky asset: $u^{9} \Phi$ must be convex ${ }^{2}$ and the relative index of risk aversion i $x u^{\oplus}(x)=u^{9}(x)$ must be bounded from above by 1 . This somewhat counter-intuitive result, that has puzzled many economists, has lead to various attempts to restrict not only the type of risk aversion postulated, but also the notion of increase in risk itself. In one of these contributions, Quiggin [21] showed that an EU DM with DARA (Decreasing Absolute Risk Aversion) utility function will reduce the allocation to the risky asset if it is subjected to a M-MPIR . It should be noted that the CARA utility function $\mathrm{i}$ expf $\mathrm{K} x \mathrm{x}$, a special case of DARA, has unbounded relative index of risk aversion, and as such, it does not meet the R othschild \& Stiglitz (1971) necessary conditions. Hence, a EU DM with CARA utility function will

\footnotetext{
${ }^{2} \mathrm{u}^{\prime}$ convex is termed "prudence" by Kimball [16].
} 
coherently reduce the allocation on a monotonely riskier asset while failing to do so under the standard notion of riskier asset.

\section{The RDEU model}

Variants of the Rank Dependent Expected Utility theory due to Quiggin [20] have been treated by Yaari [32], Chew, Karni \& Safra [11], Segal [28, 29] and Allais [2]. General axiomatization can be found in Wakker [30], Quiggin \& Wakker [22], Chateauneuf [6].

De- nition 5 A DM behaves in accordance with the Rank Dependent Expected U tility model (RDEU) if the DM is characterized by a continuous, strictly increasing utility function $u$ in conjunction with a probability-perception function $f:[0 ; 1] ![0 ; 1]$ that is strictly increasing and satis ${ }^{-}$es $f(0)=0 ; f(1)=1$. Such a $D M$ (weakly) prefers the random variable $X$ to the random variable $Y$ if and only if $V(X), V(Y)$, where the RDEU functional $V$ is given by

$$
\begin{aligned}
V(X) & =V_{u ; f}(X)=i_{i 1}^{Z_{1}} u(x) d(P(X>x)) \\
& =Z_{0}[f(P(u(X)>t)) i 1] d t+Z_{1} f(P(u(X)>t)) d t:
\end{aligned}
$$

If the support of the random variable $X$ is a " nite set, $V(X)$ can be written as

$$
\begin{aligned}
& V(X)=\sum_{i=1}^{n} u\left(x_{i}\right) 4 f\left(\sum_{j=i}^{2} p\right) i f\left(x_{j=i+1}^{n} p_{j}\right)^{3} \\
& =u\left(x_{1}\right)+x_{i=2}^{n}\left[u\left(x_{i}\right) i u\left(x_{i j}\right)\right] f\left(x_{j=i}^{n} p\right) \\
& =u\left(x_{1}\right)+x_{i=2}^{n}\left[u\left(x_{i}\right) i u\left(x_{i} 1\right)\right] f\left(v_{i j}\right) \text {; }
\end{aligned}
$$

where $v_{i}=P_{j=i+1}^{n} p=P\left(X>x_{i}\right)$.

If the perception function $f$ is the identity function $f(v)^{\prime}, v$, then $V(X)=V_{u ; I}(X)$ is the expected utility $E[u(X)]$ of the random variable. The Yari functional (see [32]) is the special case $V(X)=V_{1 ; f}(X)$, in which the utility on outcomes is the identity function $\mathrm{u}(\mathrm{x})^{\prime} \mathrm{X}$. If both perception and utility are identity functions, then $\mathrm{V}(\mathrm{X})=\mathrm{V}_{\mathrm{l} ; \mathrm{I}}(\mathrm{X})$ is simply the expected value $E[X]$ of the random variable.

In some sharp sense, M -MPIR plays for Dual Theory (in fact, for the RDEU model with concave utility) the role played by MPIR for the EU model: for equal \{mean $X$ and $Y$ in 
$V$, all RDEU DM's with linear $u$ and pessimistic $f(f(p) \cdot p)$ prefer $X$ to $Y$, if and only if $Y$ is a M-MPIR with respect to $X$ (see $[5,9]$ and implicitly [32]).

Example 1. Spreading out a two-point distribution \{ a necessary condition for monotone risk aversion.

Let $0<v<1$ and i $1<x_{1}<x_{2}<x_{3}<1$, let $x_{4}=x_{3}+\left(x_{2} i x_{1}\right)(1 ; v) \Rightarrow$. Consider the M-MPIR from the two-point distribution $L(X)=\left(x_{2} ; 1 ; v ; x_{3} ; v\right)$ (see (3)) to the twopoint distribution $L(Y)=\left(x_{1} ; 1 ; V ; X_{4} ; v\right)$. Since $E(X)=E(Y)$; monotone risk aversion implies that $V(X), V(Y)$ for all choices of $v$ and $x_{i}$ as above. Using the representation $V(X)=u\left(x_{2}\right)+f(v)\left[u\left(x_{3}\right) ; u\left(x_{2}\right)\right]$ (see (5)), this inequal ity is readily seen to be equivalent to

$$
\frac{u\left(x_{4}\right) \text { i } u\left(x_{3}\right)}{x_{4} \text { i } x_{3}}=\frac{u\left(x_{2}\right) \text { i } u\left(x_{1}\right)}{x_{2} \text { i } x_{1}} \cdot \frac{1 ; f(v)}{1 \text { i } v}=\frac{f(v)}{v}:
$$

Hence, a necessary condition for monotone risk aversion is that the supremum of the left-hand side of (6), related to the index of grediness $G_{u}$ (sœ (2)) of the utility function $u$, be less than or equal to the in ${ }^{-}$mum of the right-hand side of (6), the index of pessimism $P_{f}$ (see (1)) of the probability perception function $f$. However, the supremum of the LHS of (6) should be taken over vectors $\left(\mathrm{x}_{1} ; \mathrm{x}_{2} ; \mathrm{x}_{3} ; \mathrm{x}_{4}\right)$ satisfying $\mathrm{x}_{1}<\mathrm{x}_{2}<\mathrm{x}_{3}<\mathrm{x}_{4}$ and also $x_{4}=x_{3}+\left(x_{2} i \quad x_{1}\right)(1 ; v) \Rightarrow$, so this supremum could in principle depend on $v$. By the following Lemma 1 , this supremum, independent of $v$, is equal to the index of greediness $G_{u}$ of the utility function $u$, to be compared with the index of pessimism $P_{f}$ of the probability perception function $\mathrm{f}$.

Lemma 1 Let $u: R$ ! $R$ be continuous and strictly increasing and let, $>0$. Denote

$$
\begin{aligned}
E_{0} & =f \bar{x} j \bar{x}=\left(x_{1} ; x_{2} ; x_{3} ; x_{4}\right) 2 R^{4} ; x_{1}<x_{2} \cdot x_{3}<x_{4} ; \frac{x_{4} i x_{3}}{x_{2} i x_{1}}=, g \\
G_{u}(,) & =\sup _{\bar{x} 2 E}\left[\frac{u\left(x_{4}\right) i u\left(x_{3}\right)}{x_{4} i x_{3}}=\frac{u\left(x_{2}\right) i u\left(x_{1}\right)}{x_{2} i x_{1}}\right]:
\end{aligned}
$$

Then $G_{u}()=,G_{u}$ :

The lemma is proved in the A ppendix. As noted following the de nition of the index of non-concavity, if $u$ is di Rerentiable, then $G_{u}$ is expressible as $G_{u}=\sup _{y<x} \frac{u^{0}(x)}{u^{0}(y)}$ and Lemma 1 is super ${ }^{\circ}$ uous.

The next subsections introduce more formally these indices of pessimism and greediness as well as their dual of optimism and thriftiness (or non-convexity), and analyze some of their main properties. 


\subsection{Index of pessimism}

A RDEU DM is pessimistic under risk if the DM assesses lotteries as a EU DM who, for each $\times 2 R$; undervalues the probability to win at least $x$; this is the eßect of a probability perception function $f$ such that $f(v) \cdot v$ for all $v 2[0 ; 1]$. For any probability perception function $f$ lying under the identity function, $1 ; f(v), 1_{i} v$ and $f(v) \cdot v$. Thus, by (1), the index of pessimism $P_{f}=\inf _{0<v<1}\left[\frac{1_{i} f(v)}{1_{i} v}=\frac{f(v)}{v}\right]$ of such an $f$ satis ${ }^{-} e s P_{f}$, 1. For a probability $v$ of winning, $(1 ; \quad v) \Rightarrow$ is the odds-ratio against winning. Pessimists exaggerate this odds ratio by amplifying it to $\left(1_{i} f(v)\right) f(v)$. The index of pessimism can be intuitively understood as the minimal such ampli- cation factor.

Example 2. K ink perception function. Let a kink function be the pointwise maximum of two increasing linear functions de- ned on [0; 1]: one with slopeless than 1 going through $(0 ; 0)$, the other with slope exceeding 1 going through $(1 ; 1)$. If $f$ is a kink, then

$$
P_{f}=\min (1=f(0) ; f q(1))>1:
$$

Example 3. Hyperbolic perception function. For $P>1$, let the function

$$
f_{P}(v)=\frac{v}{v+(1 ; v) P}
$$

be called the hyperbolic perception function with index of pessimism $P$. It is an isopessimistic perception function in the sense that the expression $\left.\left[\left(1_{i} f_{P}(v)\right)=1_{i} v\right)\right]=\left[f_{P}(v)=v\right]$, whose in ${ }^{-}$mum over $v$ generally de nes theindex of pessimism, is identically equal to the index of pessimism $P$ of $f_{P}$.

The following proposition summarizes some basic properties of the index of pessimism $P_{f}$. Properties (i)-(v) are of intrinsic interest. Property (vi) is a technical lemma needed in the sequel.

\section{Proposition 2}

(i) For perception functions $f$ and $g$, if $f(v) \cdot g(v)$ for all $v 2(0 ; 1)$, then $P_{f}, P_{g}$.

(ii) If $f$ is a non-linear, convex perception function, then $P_{f}>1$.

The following property characterizes strict pessimism.

(iii) For a perception function $f, P_{f}>1$ if and only if $f$ is majorized by some nonlinear, convex perception function. Furthermore, every perception function $f$ with $P_{f}>1$ 
is majorized by the hyperbolic perception function with the same in dex of pessimism $\mathrm{P}_{f}$ ( see (9)).

(iv) Fix $P>1$ and let $L_{P}(v)=v=P$ and $R_{P}(v)=1+\left(v_{i} 1\right) P$ be the linear support functions of the hyperbolic perception function $f_{P}$ at 0 and 1 respectively. Then $P_{f}=P$ for every perception function $f$ satisfying $L_{P} \cdot f \cdot f_{P}$ or $R_{P} \cdot f \cdot f_{P}$.

The following property gives an alternative de ${ }^{-}$nition of $P_{f}$.

(v) For a pessimistic perception function $f, P_{f}=Q$, where

$$
\begin{aligned}
Q & =\operatorname{inffP}>1 j f(v)>f_{P}(v) \text { for some } v 2(0 ; 1) g \\
& =\operatorname{supfP}, \quad \operatorname{ljf}(v) \cdot f_{P}(v) \text { for all } v 2(0 ; 1) g:
\end{aligned}
$$

(vi) The index of pessimism of a pessimistic DM satis ${ }^{-}$es

$$
P_{f}=\inf _{0<v_{2} \cdot v_{1}<1} \frac{1 ; f\left(v_{1}\right)}{1 ; v_{1}}=\frac{f\left(v_{2}\right)}{v_{2}}:
$$

The proof is in the Appendix. This proposition further justi ${ }^{-} e P_{f}$ as an index of pessimism \{ the moref I hangs down" below the diagonal, the more pessimistic the DM is, and the larger $P_{f}$ is. The index of pessimism is the same for all perception functions that are $\backslash$ sandwiched" between an hyperbolic perception function and the kink that supports it from below at 0 and at 1 .

Example 4. Power-type perception functions. For ${ }^{\circledR}>1$, the perception functions $f(v)=v^{\circledR}$ and $g(v)=1 ; \quad(1 ; v)^{1=\circledR}$ have index of pessimism ${ }^{\circledR}$.

Sketch of proof: By property (iv), $f$ is sandwiched between $R_{\circledast}$ and $f_{\circledast}$, while $g$ is sandwiched between $L_{\circledast}$ and $f_{\circledast}$. The details are skipped.

Optimism and index of optimism

By duality, a RDEU DM with a probability perception function $f$ is optimistic under risk if $f(v), v$ for all $v 2[0 ; 1]$ with an index of optimism $O_{f}$

$$
O_{f}=\inf _{0<v<1}\left[\frac{f(v)}{1 ; f(v)}=\frac{v}{1 i v}\right]=\inf _{0<v<1}\left[\frac{f(v)}{v}=\frac{1 ; f(v)}{1 ; v}\right]
$$

where $\mathrm{O}_{\mathrm{f}}, 1$ for an optimist.

\subsection{Index of non-concavity (or greediness)}

For a strictly increasing utility function $u,(2)$ de- nes its index of non-concavity. Since non-decreasing functions are di ßerentiable almost everywhere, the ratio in (2) can be made 
arbitrarily close to 1 by concentrating all four points $x_{i}$ close to a point of di ßerentiability of $u$. Hence, $G_{u}$, 1. The value 1 corresponds exclusively to concavity. For di Rerentiable $u$ the index of non-concavity can be expressed in the simpler form

$$
G_{u}=\sup _{y<x} \frac{u^{Q}(x)}{u^{Q}(y)}
$$

Intuitively, as the maximal possible ampli ${ }^{-}$cation factor of marginal utility from a low level of wealth to a higher level of wealth, it measures Igreed" - valuing an additional cent more when rich than when poor. We propose to call it index of greediness.

A function $u^{\alpha}$ is said to be more concave than a function $u$ if there is a concave, strictly increasing function 'such that for all $x 2 R, u^{\mathbb{a}}(x)={ }^{\prime}(u(x))$. In this $\mathrm{de}^{-}$nition, neither of the two utility functions is required to be concave ${ }^{3}$. Since

$$
\begin{aligned}
& \frac{u^{x}\left(x_{4}\right) \text { i } u^{x}\left(x_{3}\right)}{x_{4} \text { i } x_{3}}=\frac{u^{x}\left(x_{2}\right) \text { i } u^{x}\left(x_{1}\right)}{x_{2} \text { i } x_{1}}=\frac{{ }^{\prime}\left(u\left(x_{4}\right)\right) \text { i }{ }^{\prime}\left(u\left(x_{3}\right)\right)}{x_{4} \text { i } x_{3}}=\frac{{ }^{\prime}\left(u\left(x_{2}\right)\right) \text { i }{ }^{\prime}\left(u\left(x_{1}\right)\right)}{x_{2} \text { i } x_{1}} \\
& =\left[\frac{{ }^{\prime}\left(u\left(x_{4}\right)\right) i^{\prime}\left(u\left(x_{3}\right)\right)}{u\left(x_{4}\right) \text { i } u\left(x_{3}\right)}=\frac{{ }^{\prime}\left(u\left(x_{2}\right)\right) \text { i }{ }^{\prime}\left(u\left(x_{1}\right)\right)}{u\left(x_{2}\right) \text { i } u\left(x_{1}\right)}\right]\left[\frac{u\left(x_{4}\right) \text { i } u\left(x_{3}\right)}{x_{4} \text { i } x_{3}}=\frac{u\left(x_{2}\right) \text { i } u\left(x_{1}\right)}{x_{2} \text { i } x_{1}}\right] \\
& \text { (15) } \cdot \frac{u\left(x_{4}\right) \text { i } u\left(x_{3}\right)}{x_{4} \text { i } x_{3}}=\frac{u\left(x_{2}\right) \text { i } u\left(x_{1}\right)}{x_{2} \text { i } x_{1}} \text {; }
\end{aligned}
$$

it follows directly from the de nition (see (2)) of the index of greediness that $G_{u^{x}} \cdot G_{u}$.

Proposition 3 summarizes the above properties of this index:

Proposition 3 (i) $\mathrm{G}_{\mathrm{u}}, 1$ :

(ii) $G_{u}=1$ if and only if $u$ is concave.

(iii) If $u$ is di ßerentiable,

$$
G_{u}=\sup _{y<x} \frac{u^{9}(x)}{u^{9}(y)}:
$$

(iv) If the utility function $u^{\alpha}$ is more concave than the utility function $u$, then $G_{u^{\alpha}} \cdot G_{u}$. Index of non-convexity (or thriftiness)

By duality, we can de ${ }^{-}$ne, for a strictly increasing utility function u; an index of nonconvexity (or thriftiness):

$$
\mathrm{T}_{\mathrm{u}}=\sup _{\mathrm{x}_{1}<\mathrm{x}_{2} \cdot \mathrm{x}_{3}<\mathrm{x}_{4}}\left[\frac{\mathrm{u}\left(\mathrm{x}_{2}\right) ; \mathrm{u}\left(\mathrm{x}_{1}\right)}{\mathrm{x}_{2} \mathrm{i} \mathrm{x}_{1}}=\frac{u\left(\mathrm{x}_{4}\right) ; \mathrm{u}\left(\mathrm{x}_{3}\right)}{\mathrm{x}_{4} \mathrm{i} \mathrm{x}_{3}}\right]:
$$

$\mathrm{T}_{\mathrm{u}}, 1$ and the value 1 corresponds exclusively to convexity.

\footnotetext{
${ }^{3}$ In EU theory, the utility function $\mathrm{u}^{\alpha}$ is then said to display (Arrow-P ratt) more risk aversion than the utility function $\mathrm{u}$ :
} 
Moreover, for a di Rerentiable $u$ the index of non-convexity can be expressed in the simpler form

$$
T_{u}=\sup _{x<y} \frac{u^{q}(x)}{u^{q}(y)}
$$

\section{Characterization of monotone risk aversion}

We can now state the main result:

\subsection{Main result}

Theorem 1 A RDEU DM with probability perception function $f$ and utility function $u$ is monotone risk averse if and only if the DM 's index of pessimism exceeds the DM 's index of non-concavity: $P_{f}, G_{u}$.

The theorem will be proved in Section 5. Let us ${ }^{-}$rst emphasize some implications of this result.

1. Since $G_{u}, 1$, the fact that $P_{f}, G_{u}$ for monotone risk averse $D M$ 's, implies that $P_{f}, 1$, or $f(v) \cdot v$. In other words, pessimism is a necessary condition for monotone risk aversion. This fact also follows from (i) monotone risk aversion implies weak risk aversion and (ii) weak risk aversion implies pessimism: $f(v) \cdot v$ for $v 2$ [0; 1] (se for instance Chateauneuf \& Cohen [7]).

2. If $f$ is the identity function then $P_{f}=1$. However, there are pessimistic $f$ other than the identity function itself, for which $P_{f}=1$ : it su \pm ces that $f(p)=p$ for some $p 2(0 ; 1)$ or that $f(p)=1$ at either $p=0$ or $p=1$. Thus, concavity of $u$ is necessary for monotone risk aversion only for EU maximizers and for RDEU DM \local ly close" to being EU maximizers, in this precise sense.

3. Quiggin (see [21]) uses the concept of monotone risk aversion only for DM with concave $u$. Theorem 1 proves that concavity of $u$ is not a necessary condition for monotone risk aversion. Similarly, convexity is not necessary for monotone risk-seeking attitudes (see the following corollary).

4. If $\mathrm{f}$ crosses the diagonal, the DM is neither monotone risk averse nor monotone risk seking. In fact, not even weakly so.

The following theorem is presented as a corollary since its proof is analogous to that of Theorem 1. 
Corollary 1 A RDEU DM with probability perception function $f$ and utility function $u$ is monotone risk seeking if and only if the DM's index of optimism exceeds the DM's index of non-convexity: $\mathrm{O}_{\mathrm{f}}, \mathrm{T}_{\mathrm{u}}$.

\subsection{Examples and discussion}

\subsubsection{Risk-seeking attitude with diminishing marginal utility}

Restrict attention to random variables with values in $[0 ; 1]$ and assume accordingly that the utility function is de- ned in the unit interval.

Example 5. CARA ${ }^{4}$ utility and power-type perception functions. Let a RDEU DM 's choices among lotteries on $[0 ; 1]$ be characterized by the concave CA R A utility function $u(x)=i$ ae ${ }^{b x}$ and the optimistic power-type perception function $f(v)=1_{i}\left(1_{i} v\right)^{h}$, with $a>0, b>0$ and $h>e^{b}$. As is easy to ascertain, $O_{f}=h$ and $T_{u}=e^{b}$. Hence, in spite of the concavity of the utility function, the DM is monotone risk seeking.

Note, however, that a RDEU DM with the same utility function $u(x)=i$ ae $b x$ as above but pessimistic power-type probability perception function $f(v)=v^{k}$ with $k, 1$, is monotone risk averse, since $P_{f}=k$ and $G_{u}=1$.

In summary, a DM with a given concave utility function can be monotone risk averse or monotone risk seeking or in fact neither, depending on the probability perception function.

\subsubsection{M ean-preserving increase in risk does not imply monotone mean-preserving increase in risk.}

In thefollowing example, $Y$ is a mean-preserving spread but not a monotone mean-preserving spread of $X$, because $X$ and $Z$ are not comonotone.

Example 6. A non-monotone mean-preserving spread. Consider four equally likely points and two random variables $Y$ and $X$ with respective values

$\begin{array}{lllll}Y & 0 & 1000 & 3000 & 4000 \\ X & 0 & 2000 & 2000 & 4000\end{array}$

and di ßerence $Z=Y$; X

$$
\text { Z } 0 \text { i } 1000 \quad 1000 \quad 0
$$

A ny RDEU DM with linear $u\left(G_{u}=1\right)$ and pessimistic $f\left(P_{f}, 1\right)$ is monotone risk averse because $P_{f}, G_{u}$. For any $f$ satisfying moreover $f(1=2)>\frac{f(1=4)+f(3=4)}{2}$, it is easy to see that $\mathrm{V}(\mathrm{Y})>\mathrm{V}(\mathrm{X})$ and thus this monotone risk averse $\mathrm{DM}$ strictly prefers the more

\footnotetext{
${ }^{4}$ b orrowing EU terminology that may be inappropriate beyond EU
} 
risky random variable $Y$. By Chew, Karni \& Safra [11], this could not have been possible with a convex $f$.

\subsubsection{Comparison of monotone risk aversion with weak and strong risk aversion}

As expressed in the Introduction, these three kinds of risk aversion are logically related so that strong r.a. implies monotone r.a., which in turn implies weak r.a. The three are equivalent in EU theory. In RDEU theory, pessimism is necessary for each of the three and concavity of $u$ is necessary only for strong r.a. Furthermore, the three hold simultaneously only in the class of DM 's with concave $u$ and convex $f$ (Chew, Karni $\&$ Safra [11]). In the class of DM 's with concave $u$, weak and monotone r.a. are equivalent and hold as long as $f$ displays pessimism (Q uiggin [20,21]), but do not imply strong r.a. However, if $u$ is allowed not to be concave, weak r.a. does not imply monotone r.a., as the following example shows, which we present without proof: a DM with $u(x)=x^{2}$ on $[0 ; 1)$ and $f(p)=p^{2}$, easily seen not to be monotone risk averse because $P_{f}=2$ and $G_{u}=1$, is weakly risk averse ([10], in preparation).

There is, thus, an essential di Berence in the attitude of RDEU DM's to the Quiggin B ickel \& Lehmann and Rothschild \& Stiglitz notions of increase in risk: for the latter, there is no degree of pessimism that can compensate for departures from concavity of $u$ and still make the DM averse to risk. The main theorem of this paper shows that for the stronger monotone notion of risk (and thus, weaker notion of risk aversion), such a compensation exists and is completely separable in the sense that no joint condition in terms of both $u$ and $f$ is needed. Rather, the compensation is based on a comparison of intrinsic indices of pure type, one for greediness displayed by $u$ and the other for pessimism displayed by $f$.

\section{Proof of Theorem 1}

\subsection{Necessity of the condition $P_{f}, G_{u}$}

Consider the setup introduced in Example 1, for some given $v 2(0 ; 1)$. By Lemma 1 , it is enough to prove that for every $\left(\mathrm{y}_{1} ; \mathrm{y}_{2} ; \mathrm{y}_{3} ; \mathrm{y}_{4}\right) 2 \mathrm{E}$, where, $=(1 \mathrm{i} v) \Rightarrow$, a monotone risk averse DM characterized by $(u ; f)$ veri ${ }^{-}$es

$$
\frac{u\left(y_{4}\right) i u\left(y_{3}\right)}{y_{4} i y_{3}}=\frac{u\left(y_{2}\right) ; u\left(y_{1}\right)}{y_{2} i y_{1}} \cdot \frac{1 ; f(v)}{1 ; v}=\frac{f(v)}{v}:
$$

Let a random variable $\mathrm{X}$ have values $\mathrm{y}_{2}<\mathrm{y}_{3}$ and respective probabilities $1 \mathrm{i} v$ and $\mathrm{v}$. 
The RDEU functional for a DM characterized by a pair ( $u ; f$ ) is (see (5))

$$
V(X)=u\left(y_{2}\right)+f(v)\left[u\left(y_{3}\right) ; u\left(y_{2}\right)\right]:
$$

Let us apply a monotone mean-preserving spread to this distribution in the following way: Since $\left(\mathrm{y}_{1} ; \mathrm{y}_{2} ; \mathrm{y}_{3} ; \mathrm{y}_{4}\right) 2 \mathrm{E}$, there exists ${ }^{\circ}>0$ such that $\mathrm{y}_{1}=\mathrm{y}_{2} \mathrm{i}^{\circ} \mathrm{v}$ and $\mathrm{y}_{4}=\mathrm{y}_{3}+{ }^{\circ}\left(1_{\mathrm{i}} \mathrm{v}\right)$. The random variable $\mathrm{Y}$ taking values $\mathrm{y}_{1}<\mathrm{y}_{4}$ with respective probabilities $1 \mathrm{i} v$ and $v$ is then riskier than $X$ in the sense of a M-M PIR. Since

$$
V(Y)=u\left(y_{1}\right)+f(v)\left[u\left(y_{4}\right) ; u\left(y_{1}\right)\right]
$$

then $V(Y)$ i $V(X) \cdot 0$ if and only if $(1 ; f(v))\left(u\left(y_{1}\right)\right.$ i $\left.u\left(y_{2}\right)\right)+f(v)\left(u\left(y_{4}\right)\right.$ i $\left.u\left(y_{3}\right)\right) \cdot 0$ But $\left(y_{4}\right.$ i $\left.y_{3}\right) \neq y_{2}$ i $\left.y_{1}\right)=(1 ; \quad v) \Rightarrow$, so the required inequality (19) follows.

\subsection{Sut ciency of the condition $P_{f}, G_{u}$}

We must compare $\mathrm{V}(\mathrm{X})$ and $\mathrm{V}(\mathrm{Y})$ for an arbitrary pair $(\mathrm{X} ; \mathrm{Y})$ of (equal-mean) random variables for which $Y$ is a M-MPIR with respect to $X$. The proof will proceed in two steps: (i) su \pm ciency of the condition for random variables with - nitely many values, and (ii) a continuity argument to extend su \pm ciency of the condition to the set $V$, composed of bounded random variables in general.

\subsubsection{The discrete case}

As in Landsberger \& M eilijson [18] and Chateauneuf, Cohen \& Meilijson [9], let the result of an out-stretch $(v, w ; a, b)$ (where $0<v \cdot w<1$ and $a ; b>0$ ) of a distribution function $F$ be the distribution $\mathrm{G}$ obtained from $\mathrm{F}$ by shifting to the right by an amount bthe section of $F$ above height $1_{i} v$ and by a to the left the section of $F$ below height $1_{i}$ w. (If $F$ is an income distribution, then the poorest $(1 ; w)$-quantile of the population becomes poorer by the constant amount $\mathrm{a}$ and the richest v-quantile becomes richer by the constant amount b). Clearly, if $v b=(1 ; w) a$, then $(v ; w ; a ; b)$ is a mean-preserving out-stretch. Notice that these out-stretches may entail splitting an atom in each side in two, one part of which gets shifted and the other stays in place. It is intuitively true and easy to prove (see [18]) that (i) if $G$ is obtained from $F$ by a mean-preserving out-stretch then $G$ is a M-MPIR with respect to $F$, and (ii) if $F$ and $G$ are supported by - nite sets and $G$ is a M-MPIR with respect to $F$, then there is a ${ }^{-}$nite sequence $F=F_{1} ; F_{2} ;:: ; F_{k}=G$ of distributions such that for each $1 \cdot i<k, F_{i+1}$ is a mean-preserving out-stretch of $F_{i}$. Due to these facts, it 
is enough to prove that $\mathrm{V}(\mathrm{Y}) \cdot \mathrm{V}(\mathrm{X})$ whenever the distribution of $\mathrm{Y}$ is a mean-preserving out-stretch of that of $X$. Let $(v ; w ; a ; b)$ be this stretch and let

$$
\begin{aligned}
L(X)= & \left(x_{1} ; 1 \text { i } v_{1} ; x_{2} ; v_{1} \text { i } v_{2} ;:: ; ; x_{i} ; v_{i j} 1 \text { i } v_{i} ;::: ; x_{j} ; v_{j i} 1 \text { i } v_{j}\right. \\
& \left.::: ; x_{n_{i} 1} ; v_{n_{i}} 2 \text { i } v_{n_{i}} ; x_{n} ; v_{n_{i} 1}\right)
\end{aligned}
$$

bethe distribution of $X$, with atoms $x_{1}<x_{2}<\Phi \pitchfork \Varangle<X_{n}$ and probabilities $P\left(X>X_{k}\right)=v_{k}$. Let $i<j$ be chosen so that $v_{i} 1, w, v_{i}, v_{j i}, v, v_{j}$. Then $V(X) i V(Y)$ is

$$
\begin{aligned}
& \$ V=\left[u\left(x_{1}\right) ; u\left(x_{1} ; a\right)\right] f 1 ; f\left(v_{1}\right) g+\left[u\left(x_{2}\right) ; u\left(x_{2} ; a\right)\right] f f\left(v_{1}\right) ; f\left(v_{2}\right) g+\$ \nsubseteq \varnothing \\
& +\left[u\left(x_{i}\right) ; u\left(x_{i} ; a\right)\right] f f\left(v_{i j 1}\right) ; f(w) g_{i}\left[u\left(x_{j}+b\right) ; u\left(x_{j}\right)\right] f f(v) ; f\left(v_{j}\right) g \\
& \text { i }\left[u\left(x_{j+1}+b\right) ; u\left(x_{j+1}\right)\right] f f\left(v_{j}\right) \text { i } f\left(v_{j+1}\right) g_{i} \pitchfork \Varangle \Phi_{i}\left[u\left(x_{n}+b\right) ; u\left(x_{n}\right)\right] f f\left(v_{n_{i}}\right) g \text { : }
\end{aligned}
$$

To prove non-negativity of (23), it is enough to prove its non-negativity when each term in square brackets with a + sign in front is replaced by the minimum of these terms, and each term in square brackets with a $\mathrm{i}$ sign is replaced by the maximum of these. Let the minimum of $u\left(x_{k}\right) i u\left(x_{k} i\right.$ a) over $k$ - i be attained at $k=1$ and the maximum of $u\left(x_{k}+b\right)$ i $u\left(x_{k}\right)$ over $k$, j i 1 be attained at $k=m$. Then, since $(1 ; w) a=v b$,

$$
\begin{aligned}
& \$ V, \quad\left[y_{1}\left(x_{l}\right) \text {; } u\left(x_{l} \text { i a }\right)\right] f 1 \text { i } f(w) g_{\text {i }}\left[u\left(x_{m}+b\right) \text { i } u\left(x_{m}\right)\right] f f(y) g \\
& =\frac{u\left(x_{1}\right) ; u\left(x_{1} ; a\right)}{a} \frac{1 ; f(w)}{1 ; w} ; \frac{u\left(x_{m}+b\right) ; u\left(x_{m}\right)}{b} \frac{f(v)}{v} v b \\
& \text {, } \frac{u\left(x_{1}\right) \text { i } u\left(x_{1} \text { i a }\right)}{a} \frac{f(v)}{v} f\left[\frac{1 ; f(w)}{1 ; w}=\frac{f(v)}{v}\right] ; G_{u} g v b \text { : }
\end{aligned}
$$

Since by assumption $P_{f}, G_{u}$, the last term is non-negative by Proposition 2 (vi). This completes the proof of su \pm ciency of the condition $P_{f}, G_{u}$, dealing with random variables supported by a - nite set. It remains to present a continuity argument that will extend su \pm ciency to general bounded random variables.

\subsubsection{Continuity arguments for the general case}

Let us now consider two elements $X$ and $Y$ of $V$, such that $Y$ is a M-MPIR of $X$. We can assume without loss of generality that $Y=X+Z$ with $Z$ and $X$ comonotone and $E(Z)=0$.

We use the standard uniform approximations of bounded random variables by nondecreasing step functions, which preserve comonotonicity.

Thus, let $X_{n}$ and $Z_{n}$ be such approximations of $X$ and $Z$. Explicitly,

$$
X_{n}=\sum_{i=0}^{n \times n}\left[\frac{i}{2^{n}} I_{f} \frac{i}{2^{n}} \cdot x<\frac{i+1}{2^{n}} g i \frac{i+1}{2^{n}} I_{f i} \frac{i+1}{2^{n}} \cdot x<i \frac{i}{2^{n}} g^{]}\right.
$$


where $1_{A}$ denotes the characteristic function of the event $A$. A similar expression de nes $Z_{n}$. $X_{n}$ and $Z_{n}$ are comonotone, since they are non-decreasing functions of comonotone random variables. (One of the equivalent de $e^{-}$nitions of comonotonicity of $X$ and $Z$ is the existence of a random variable $U$ of which each of $X$ and $Z$ is a non-decreasing function. Obviously, so are $X_{n}$ and $Z_{n}$, automatically).

For $n$ large enough so that $X ; Y$ and $Z$ are supported by the interval (i $n ; n)$,

$$
X i \frac{1}{2^{n}} \cdot X_{n} \cdot X \text { and } Z i \frac{1}{2^{n}} \cdot Z_{n} \cdot Z \text { : }
$$

Hence, with $Y_{n}=X_{n}+Z_{n}$,

$$
Y_{i} 12^{n i 1} \cdot Y_{n} \cdot Y:
$$

The second statement in (26) and the fact that $E(Z)=0$ entail $E\left(Z_{n}\right)$ " 0 . De ne then $\mathbb{R}_{\mathrm{n}} \# 0$ by $E\left(Z_{\mathrm{n}}+\mathbb{R}_{\mathrm{n}}\right)=0$ and set $Z_{\mathrm{n}}^{0}=Z_{\mathrm{n}}+\mathbb{R}_{\mathrm{n}} ; Y_{\mathrm{n}}^{0}=X_{\mathrm{n}}+Z_{\mathrm{n}}^{0}$ :

Clearly, $X_{n}$ and $Y_{n}^{0}$ are ${ }^{-}$nite-support random variables and $Y_{n}^{0}$ has been obtained from $X_{n}$ by a M-MPIR. It follows from part (i) of the proof that for each $n$,

$$
\mathrm{V}\left(\mathrm{X}_{\mathrm{n}}\right), \mathrm{V}\left(\mathrm{Y}_{\mathrm{n}}^{0}\right):
$$

For a random variable $T, V(T)$ is the Choquet integral of $u(T)$ with respect to the capacity $c=f \pm P$, i.e., $V(T)={ }^{R} u(T)$ f $\pm P$. Since the Choquet integral is monotone and comonotonically additive ( $s e[5,13,26,27]$ ), (28) will entail the desired result $V(X)$, $\mathrm{V}(\mathrm{Y})$. F or the sake of completeness, we present a direct argument.

$Y_{n}^{0}, Y_{n}$ implies $u\left(Y_{n}\right), u\left(Y_{n}\right)$ and, a-fortiori, $V\left(Y_{n}^{0}\right), V\left(Y_{n}\right)$. By (28),

$$
\mathrm{V}\left(\mathrm{X}_{\mathrm{n}}\right), \mathrm{V}\left(\mathrm{Y}_{\mathrm{n}}\right) ; 8 \mathrm{n} 2 \mathrm{~N}:
$$

Since the utility function $u$ is continuous, it is uniformly continuous on any compact subset of R. Hence, from (26) and (27), $8^{2} 9 \mathrm{~N}\left({ }^{2}\right)$ s.t. $n, N\left({ }^{2}\right)$ implies that

$$
u(X) i^{2} \cdot u\left(X_{n}\right) \cdot u(X) ; u(Y) i^{2} \cdot u\left(Y_{n}\right) \cdot u(Y):
$$

Monotonicity, comonotonic additivity and the normalization property ${ }^{R} 1$ \& $\pm P=1$ lead to the two inequalities: $8 \mathrm{n}, \mathrm{N}\left({ }^{2}\right)$,

$$
V(X) i^{2} \cdot V\left(X_{n}\right) \cdot V(X) ; V(Y) i^{2} \cdot V\left(Y_{n}\right) \cdot V(Y):
$$

Therefore, $\lim _{n ! 1} \mathrm{~V}\left(X_{n}\right)=\mathrm{V}(X)$ and $\lim _{n ! 1} \mathrm{~V}\left(\mathrm{Y}_{\mathrm{n}}\right)=\mathrm{V}(\mathrm{Y})$. Hence, by (29), $V(X), V(Y)$. 
Remark A RDEU DM was postulated in De nition 5 to have a continuous, strictly increasing utility function $u$ and a strictly increasing probability perception function $f$. The preceding proof could be somewhat simpli ${ }^{-}$ed if we added di ${ }^{\circledR e}$ erentiability assumptions on $\mathrm{u}$.

\section{A ppendix}

\section{Proof of Lemma 1}

Obviously, $G_{u}\left(\right.$, ) $G_{u}$. We have to prove the opposite inequality $G_{u}\left(\right.$, ) , $G_{u}$, i.e., for any $\left(\mathrm{x}_{1} ; \mathrm{x}_{2} ; \mathrm{x}_{3} ; \mathrm{x}_{4}\right) 2 \mathrm{R}^{4}$ such that $\mathrm{x}_{1}<\mathrm{x}_{2} \cdot \mathrm{x}_{3}<\mathrm{x}_{4}$ and any " $>0$, there exists a $\left(y_{1} ; y_{2} ; y_{3} ; y_{4}\right) 2 E$, such that

$$
\frac{u\left(y_{4}\right) \text { i } u\left(y_{3}\right)}{y_{4} i y_{3}}=\frac{u\left(y_{2}\right) \text { i } u\left(y_{1}\right)}{y_{2} \text { i } y_{1}}, \frac{u\left(x_{4}\right) \text { i } u\left(x_{3}\right)}{x_{4} \text { i } x_{3}}=\frac{u\left(x_{2}\right) \text { i } u\left(x_{1}\right)}{x_{2} i x_{1}} i^{2}:
$$

By continuity of $u$, there exists some $x_{0} 2\left(x_{3} ; x_{4}\right)$ such that for every $\times 2\left(x_{0} ; x_{4}\right)$,

$$
\frac{u(x) \text { i } u\left(x_{3}\right)}{x_{i} x_{3}}=\frac{u\left(x_{2}\right) ; u\left(x_{1}\right)}{x_{2} i x_{1}}, \frac{u\left(x_{4}\right) ; u\left(x_{3}\right)}{x_{4} i x_{3}}=\frac{u\left(x_{2}\right) ; u\left(x_{1}\right)}{x_{2} i x_{1}} i^{2}:
$$

Divide the interval $\left(x_{1} ; x_{2}\right)$ into $K$ sub-intervals of equal length $\phi=\left(x_{2} ; x_{1}\right)=$ such that,$\$<x_{4} i x_{0}$. This guarantees that the sequence $x_{3} ; x_{3}+, \notin ; x_{3}+2, q ; x_{3}+3, \downarrow ;::$ : has some element $x_{3}+k, \phi$ (call it $\left.x\right)$ in the interval $\left(x_{0} ; x_{4}\right)$.

Since $\left(u(x)\right.$ i $\left.u\left(x_{3}\right)\right)=\left(x ; \quad x_{3}\right)=(1 \neq k) P_{i=0}^{k_{i} 1}\left[u\left(x_{3}+(i+1), \phi\right) i \quad u\left(x_{3}+i, \phi\right)\right]=$, , there is a sub-interval $\left(y_{3} ; y_{4}\right)=\left(x_{3}+i, \phi ; x_{3}+(i+1), ф\right)$ of $\left(x_{3} ; x_{4}\right)$ such that $\left(\mathrm{u}\left(\mathrm{y}_{4}\right)\right.$ i $\left.\mathrm{u}\left(\mathrm{y}_{3}\right)\right) \neq \mathrm{y}_{4}$ i $\left.\mathrm{y}_{3}\right), \quad\left(\mathrm{u}(\mathrm{x})\right.$ i $\left.u\left(\mathrm{x}_{3}\right)\right)=\left(\mathrm{x}_{\mathrm{i}} \mathrm{x}_{3}\right)$. Similarly, there exists a sub-interval $\left(y_{1} ; y_{2}\right)=\left(x_{1}+j \phi ; x_{1}+(j+1) \phi\right)$ of $\left(x_{1} ; x_{2}\right)$ along which $\left.\left(u\left(y_{2}\right) i \quad u\left(y_{1}\right)\right) \neq y_{2} i \quad y_{1}\right)$. $\left(u\left(x_{2}\right)\right.$; $\left.u\left(x_{1}\right)\right) \neq x_{2}$ i $\left.x_{1}\right)$. This completes the proof.

\section{P roof of P roposition 2}

Property (i) follows easily from the de nition of $P_{f}$ (see (1)) as the in ${ }^{-}$mum of $(v=(1 ; v))(1 f(v) ; 1))$, that majorizes the corresponding expression involving $g$.

Property (ii) follows from Property (i) by observing that every non-linear, convex perception function is separated from the identity function by some kink function (see (9).

One direction of the proof of Property (iii) is clear: If $f \cdot g$ and $g$ is a non-linear, convex perception function, then by Properties (i) and (ii), $P_{f}, P_{g}>1$. As for the 
op posite direction, assume that $P_{f}>1$. Let $\left.g(v)=f_{P_{f}}(v)=v \neq v+(1 ; v) P_{f}\right)$ be the hyperbolic perception function with index of pessimism $P_{f}$ and re-write the inequality

$$
\left.\left.\left.P_{f}=\inf _{w}[(1 ; \quad f(w)) \neq 1 ; \quad w)\right] \neq f(w) \neq v\right] g \cdot[(1 ; \quad f(v))=(1 ; \quad v)] \neq f(v) \neq v\right]
$$

simply as $f \cdot g$

P roperty (iv) is proved by ${ }^{-} r$ st applying P roperty (i) to obtain that $P_{f}, P_{f_{p}}=P$, and

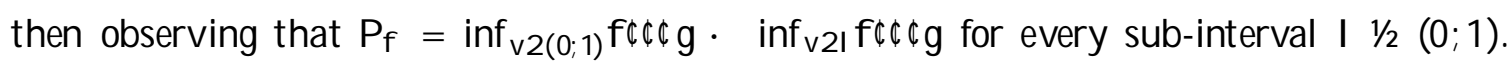
The inequality $P_{f} \cdot P$ follows by taking $I=\left(0 ;{ }^{2}\right)$ and minorizing $f$ by $L_{P}$ on $I$, or by taking $\mathrm{I}=\left(1 ;{ }^{2} ; 1\right)$ and minorizing $f$ by $R_{P}$ on $I$.

Proof of Property $(\mathrm{v})$ : To se that $\mathrm{P}_{\mathrm{f}}, \mathrm{Q}$, observe that this is trivial if $\mathrm{Q}=1$ and concentrate on the case $\mathrm{Q}>1$. Take any ${ }^{2} 2(0 ; \mathrm{Q} i 1)$. By de nition of $\mathrm{Q}, \mathrm{f}_{\mathrm{Q}_{\mathrm{i}}}{ }^{-}$majorizes

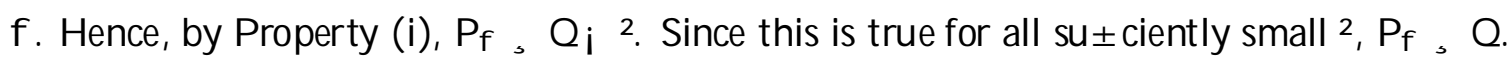
To prove the opposite inequality, take any $P>Q$ and any $v 2(0 ; 1)$ at which $f(v)>f_{P}(v)$. Then,

$$
P_{f}=\inf _{w} \frac{1 ; f(w)}{1 ; w}=\frac{f(w)}{w} \cdot \frac{1 ; f(v)}{1 ; v}=\frac{f(v)}{v}<\frac{1 ; f_{P}(v)}{1 ; v}=\frac{f_{P}(v)}{v}, P:
$$

Since $P_{f}<P$ for all $P>Q$, the inequality $P_{f} \cdot Q$ follows.

Proof of Property (vi): It is clear that the right-hand side of (12) is less than or equal $P_{f}$, since the in ${ }^{-}$mum over $v_{2} \cdot v_{1}$ is less than or equal to the in ${ }^{-}$mum over $v_{2}=v_{1}$, that is $P_{f}$ by de ${ }^{-}$nition. To see that the in ${ }^{-}$mand in the right-hand side of (12) is greater or equal than $\mathrm{P}_{\mathrm{f}}$, it is enough to show that for arbitrary $0<\mathrm{v}_{2}<\mathrm{v}_{1}<1$,

$$
\frac{1 ; f\left(v_{1}\right)}{1 ; v_{1}}=\frac{f\left(v_{2}\right)}{v_{2}}, \min \left[\frac{1 ; f\left(v_{1}\right)}{1 ; v_{1}}=\frac{f\left(v_{1}\right)}{v_{1}} ; \frac{1 ; f\left(v_{2}\right)}{1 ; v_{2}}=\frac{f\left(v_{2}\right)}{v_{2}}\right]:
$$

Otherwise, $f\left(v_{2}\right)=v_{2}>f\left(v_{1}\right)=v_{1}$ and $\left.\left(1 ; f\left(v_{2}\right)\right) \neq 1 ; v_{2}\right)>\left(1 ; f\left(v_{1}\right)\right)=1$ i $\left.v_{1}\right)$, so $f\left(v_{1}\right)>1$ i $\left.\left.\left(1 ; f\left(v_{2}\right)\right)\left(1 ; \quad v_{1}\right)=1 ; \quad v_{2}\right)>1 ; \quad\left(1 ; \quad f\left(v_{1}\right) v_{2}=v_{1}\right)\left(1 ; \quad v_{1}\right) \neq 1 ; \quad v_{2}\right)$. Extracting $f\left(v_{1}\right)$ from the ${ }^{-} r s t$ and third terms implies that $f\left(v_{1}\right)>v_{1}$, a contradiction.

\section{R eferences}

[1] Allais, M., (1953). Le comportement de l'homme rationnel devant le risque: critique des postulats et axiomes de l'cole amiricaine. E conometrica, 21, 503\{546.

[2] Allais, M., (1988). The general theory of random choices in relation to the invariant cardinal utility function and the speci ${ }^{-}$c probability function. In Risk, Decision and Rationality, B. R. M unier (Ed.), Reidel: Dordrecht. 233\{289. 
[3] Bickel, P. J. and E. L. Lehmann, (1976). Descriptive statistics for non-parametric models, III: Dispersion. A nnals of Statistics, 4, 1139\{1158.

[4] Bickel, P. J. and E. L. Lehmann, (1979). Descriptive statistics for non-parametric models, IV : Spread. In Contributions to Statistics, J. J ureckova (Ed.), Reidel: Boston, MA.

[5] Chateauneuf, A., (1994). M odeling attitudes towards uncertainty and risk through the use of Choquet integral. Annals of Operations Research, $52,3\{20$.

[6] Chateauneuf, A., (1999). Comonotonicity axioms and RDEU theory for arbitrary consequences, J ournal of Mathematical Economics, 32, 21-45.

[7] Chateauneuf, A . and M. Cohen, (1994). Risk-seeking with diminishing marginal utility in a non-expected utility model. J ournal of Risk and Uncertainty, 9, $77\{91$.

[8] Chateauneuf, A., M. Cohen and R. Kast, (1997). A review of some results related to comonotonicity. Cahiers d'E co\&M ath, 97.32.

[9] Chateauneuf, A., M. Cohen and I. Meilijson, (2003). Four notions of mean-preserving increase in risk, risk attitudes and applications to the rank-dependent expected utility model. In press, J ournal of Mathematical Economics.

[10] Chateauneuf, A., M. Cohen and I. M eilijson, (2003). Weak risk aversion and the quest for simplicity: a characterization of the preference for safety in the Rank-dependent Expected Utility model. In preparation.

[11] Chew, S., E. Karni and Z. Safra, (1987). Risk aversion in the theory of expected utility with Rank Dependent preferences. J ournal of Economic Theory, 42, $370\{381$.

[12] Cohen, M ., (1995). Risk aversion concepts in expected and non-expected utility models. The Geneva Papers on Risk and Insurance theory, 20, 73\{91.

[13] Denneberg, D., (1994). Non-additive measure and integral. K luwer Academic Publishers.

[14] Diamond, P. and J. Stiglitz, (1974). Increases in risk and risk aversion. J ournal of E conomic Theory, 8, $337\{360$. 
[15] J ewitt, I. (1989). Choosing between risky prospects:the characterisation of comparative statics results and Location Independent Risk. Management Science, 35, 60-70.

[16] K imball, M. (1990). Precautionary savings in the small and in the large. E conometrica, $58,53-73$.

[17] Landsberger, M. and I. M eilij son, (1990). Lotteries, insurance and star-shaped utility functions. J ournal of E conomic Theory, 52, $1\{17$.

[18] Landsberger, M. and I. Meilijson, (1994). The generating process and an extension of J ewitt's location independent risk concept. Management Science, 40, 662\{669.

[19] Landsberger, M. and I. M eilijson, (1994). Comonotone allocations, Bickel-Lehmann dispersion and the Arrow-Pratt measure of risk aversion. Annals of Operations Research, 52, $97\{106$.

[20] Quiggin, J., (1982). A theory of anticipated utility. J ournal of Economic Behavior and Organisation, $3,323\{343$.

[21] Quiggin, J., (1992). Increasing risk: another de- nition. In Progress in Decision, Utility and R isk Theory, A. Chikan (Ed.), Kluwer: Dordrecht.

[22] Quiggin, J. and P. P. Wakker, (1994). The axiomatic basis of anticipated utility: a clari' cation. J ournal of Economic Theory, 64, 487\{499.

[23] R oss, S., (1981). Some stronger measures of risk aversion in the small and in the large, with applications. E conometrica, 49, $621\{663$.

[24] Rothschild, M. and J. Stiglitz, (1970). Increasing Risk I: A de- nition. J ournal of E conomic Theory, 2, 225\{243.

[25] R othschild, M. and J. Stiglitz, (1971). Increasing R isk II: Its E conomic Consequences. J ournal of Economic Theory, 3, $66\{84$.

[26] Schmeidler, D., (1986). Integral representation without additivity, Proceedings of the A merican M athematical Soci ety, $97,255\{261$.

[27] Schmeidler, D., (1989). Subjective probability and expected utility without additivity. Econometrica, 57, 517\{587. First version: Subjective expected utility without additivity, Forder Institute Working Paper (1982). 
[28] Segal, U., (1989). Anticipated utility: a measure representation approach. A nnals of Operati ons Research, 19, 359\{374.

[29] Segal, U., (1993). The measure representation: A correction. J ournal of Risk and Uncertainty, 6, 99\{107.

[30] Wakker, P., (1994). Separating marginal utility and risk aversion. Theory and Decisi on, $36,1\{44$.

[31] Wilson, R., (1968). T he theory of syndicates. E conometrica, 36, 119\{132.

[32] Yaari, M., (1987). The dual theory of choice under risk. Econometrica, 55, $95\{115$. 